\title{
Correction: Aspirin for primary prevention of cardiovascular disease?
}

Our article Aspirin for primary prevention of cardiovascular disease? (DTB 2009; 47: 122-5) included a few typographical errors in the reference citations of the opening paragraphs. A corrected version of these paragraphs is available online at dtb.bmj.com. These reference errors do not affect the Conclusion of the article. 